\title{
The Role of Clinical Prediction Tools to Risk Stratify Patients with Melanoma After a Positive Sentinel Lymph Node Biopsy
}

\author{
Michael E. Egger, MD, MPH \\ Hiram C. Polk Jr., M.D. Department of Surgery, University of Louisville, Louisville, KY
}

We are currently trying to reconcile two of the greatest paradigm shifts in the treatment of melanoma in the modern era. For the first time, we have effective, safe, and well-tolerated adjuvant therapy options that improve recurrence-free survival for patients with stage III disease. $^{1-4}$ The caveat is that all of the patients in these trials were treated with a completion lymphadenectomy, and high-risk patients were selected by either eliminating patients with IIIA disease or allowing only those IIIA patients with a micrometastatic tumor burden in excess of $1 \mathrm{~mm}$. The second paradigm shift relates to the management of the nodal basin after a positive sentinel lymph node; both DeCOG-SLT and MSLT-II have shown that a completion lymphadenectomy does not improve survival in these patients. ${ }^{5,6}$ Instead, a lymphadenectomy offers only prognostic information and modest improvements in regional disease control. So how do we reconcile these two treatment paradigms? On one hand, we have safe, effective adjuvant therapy options in a well-defined, high-risk, stage III population. On the other hand, we do not know who falls into this well-defined, high-risk, stage III population, because we are no longer performing completion lymphadenectomy routinely. We are left with mostly stage IIIA patients with a single positive sentinel lymph node who need to be risk-stratified to select those who will benefit from adjuvant therapy without any additional lymph node staging. The study by Bertolli et al. ${ }^{7}$ in this issue presents a valuable tool to help risk-stratify patients using pathologic information available after sentinel lymph node biopsy.

(C) Society of Surgical Oncology 2021

First Received: 31 October 2018;

Published Online: 28 May 2021

M. E. Egger, MD, MPH

e-mail: michael.egger@louisville.edu
The Bertoli study offers a nomogram for predicting the risk of nonsentinel lymph node metastases based on three simple pathologic factors: (1) Breslow thickness, (2) micrometastatic tumor burden diameter in the sentinel lymph node, and (3) the total number of tumor-positive sentinel lymph nodes. ${ }^{7}$ The predictive model was developed in a large series of patients from the AC Camargo Cancer Center in Brazil and validated in an equally large dataset from the Netherlands Cancer Institute-Antoni van Leeuwenhoek in the Netherlands. The nomogram is simple and easy to calculate and its predictive ability was confirmed in the validation set. The study meets many of the criteria proposed by Mahar et al. that should be used when critically evaluating clinical prognostic tools for melanoma, including a clear definition of the patient population, well-defined statistical methods, and both internal and external validation assessments. ${ }^{8}$ The authors propose that the nomogram can be used to identify patients for subsequent completion lymphadenectomy if they are at high risk for nonsentinel lymph node metastases or for risk stratification and clinical decision making regarding adjuvant therapy. The latter use of the nomogram is much more compelling than the former.

Patients at high risk of nonsentinel lymph node metastases need adjuvant therapy, not additional surgery. The reasons proposed by the authors for offering a completion lymphadenectomy in high-risk patients, including inability to receive adjuvant therapy or inability to follow patients as outlined in previous trials, are reasonable but infrequent situations. We know that additional surgery only offers small improvements in regional disease control and no survival benefit. The only other benefit to lymphadenectomy is additional prognostic information, but if the nomogram predicts high risk, then the default should be to offer these patients adjuvant therapy. As we improve the accuracy of clinical prediction tools, such as the one 
offered in this article by Bertolli and colleagues, completion lymphadenectomy will only be needed in the rare cases in which definitive information is needed about the nonsentinel lymph nodes to recommend adjuvant therapy or not.

The critical population in whom tough decisions need to be made regarding adjuvant therapy are those patients with a single positive sentinel lymph node (the vast majority of patients). Those patients could fall into a number of widely varying prognostic groups. As a whole, this group has a fairly favorable prognosis, with 75\% 10-year survival reported in the most recent 8th edition International Melanoma Database for N1a patients with a single positive sentinel lymph node. ${ }^{9}$ We need prediction tools that can inform clinicians which of these patients with a single positive sentinel lymph node will fall into the IIIA group (in whom adjuvant therapy can be omitted) and those who are likely to be IIIB and IIIC and need adjuvant therapy. For those with a single positive node, this essentially becomes a question of whether they have nonsentinel lymph node metastases. Patients with a high micrometastatic disease burden, ulcerated tumors, or multiple $(>2)$ positive sentinel lymph nodes would reach the inclusion criteria for the previously mentioned pivotal adjuvant therapy trials. Thus, no nomogram or completion lymphadenectomy is needed for decision making in these patients; they should receive adjuvant therapy. I hope that in future studies, Bertolli and colleagues and others can develop accurate, validated nomograms that focus on this particular group of patients in whom there exists a real clinical dilemma regarding the need for adjuvant therapy.

It is important to note that mitotic rate was an important predictor of nonsentinel lymph node status in the Brazilian cohort when measured as a continuous variable. This factor should continue to be evaluated in other studies that seek to answer the question of how one can predict the status of the nonsentinel lymph nodes. Interestingly, ulceration was not included in the final model to predict nonsentinel lymph node status. Ulceration is certainly a risk factor for melanoma-specific survival across any cohort of patients with melanoma. Even if ulceration may lack some predictive ability regarding nonsentinel lymph nodes, when one is considering the use of adjuvant therapy, the presence of ulceration should warrant careful consideration.

As we gently step out into the untested, but promising, waters of the future of adjuvant therapy in melanoma without a completion lymphadenectomy, clinical prediction tools such as this one can be important tools to risk stratify patients and give us some confidence in our recommendations for or against adjuvant therapy. The situation should be rare in which a completion lymphadenectomy is necessary to "break the tie" and provide the final prognostic information needed to decide on adjuvant therapy. As these tools become more accurate, accounting for clinical, pathologic, and molecular risk factors, we will become more patient-specific in our approach. More accurate biomarkers or molecular signatures are needed in these patients. The next phase of research in this area will improve these prediction tools and start to tackle the much more difficult question regarding the appropriate use of adjuvant therapy in these patients, as we consider the tremendous costs associated with adjuvant therapy.

\section{REFERENCES}

1. Eggermont AM, Chiarion-Sileni V, Grob JJ, et al. Prolonged survival in stage III melanoma with ipilimumab adjuvant therapy. $N$ Engl J Med. 2016;375(19):1845-55.

2. Weber J, Mandala M, Del Vecchio M, et al. Adjuvant nivolumab versus ipilimumab in resected stage III or IV melanoma. $N$ Engl $J$ Med. 2017;377(19):1824-35.

3. Long GV, Hauschild A, Santinami M, et al. Adjuvant dabrafenib plus Trametinib in stage III BRAF-mutated melanoma. $N$ Engl $J$ Med. 2017;377(19):1813-23.

4. Eggermont AMM, Blank CU, Mandala M, et al. Adjuvant pembrolizumab versus placebo in resected stage III melanoma. $N$ Engl J Med. 2018;378(19):1789-801.

5. Leiter U, Stadler R, Mauch C, et al. Complete lymph node dissection versus no dissection in patients with sentinel lymph node biopsy positive melanoma (DeCOG-SLT): a multicentre, randomised, phase 3 trial. Lancet Oncol. 2016;17(6):757-67.

6. Faries MB, Thompson JF, Cochran AJ, et al. Completion dissection or observation for sentinel-node metastasis in melanoma. N Engl J Med. 2017;376(23):2211-22.

7. Bertolli E, Viola Franke V, Calsavara VF, et al. Validation of a nomogram for non-sentinel node positivity in melanoma patients and its clinical implications: a Brazilian-Dutch study. Ann Surg Oncol. 2019;26:395-405. https://doi.org/10.1245/s10434-018-703 $8-9$.

8. Mahar AL, Compton C, Halabi S, et al. Critical assessment of clinical prognostic tools in melanoma. Ann Surg Oncol. 2016;23(9):2753-61.

9. Gershenwald JE, Scolyer RA, Hess KR, et al. Melanoma staging: evidence-based changes in the American Joint Committee on Cancer eighth edition cancer staging manual. CA Cancer J Clin. 2017;67(6):472-92. 